\title{
Utilisation et appropriation des technologies informatiques pour l'apprentissage : cas des technicien-nes du secteur économique informel de la vente et de la réparation des appareils informatiques en Haïti
}

\author{
Valérie Payen Jean Baptiste, Université de Genève \\ Nicolas Nova, Haute-Ecole d'Art et de Design (HEAD - Genève) \\ Daniel K. Schneider, Université de Genève
}

\section{DOI : 10.51186/journals/ed.2020.10-2.e345}

\section{Résumé}

Cet article contribue à la réflexion sur l'acquisition de connaissances et de compétences en milieu informel via l'utilisation des technologies de l'information et de la communication (TIC). II présente les résultats d'une étude ethnographique portant sur les processus d'apprentissage des professionnel-les de la vente et de la réparation des téléphones mobiles en Haïti pour acquérir les connaissances et compétences nécessaires à l'exercice de leur métier. Ces processus correspondent à deux catégories : (1) les démarches d'apprentissages basées sur les manipulations pour comprendre et faire fonctionner les appareils, et (2) celles qui incluent les processus d'appropriation et de réutilisation des appareils. Le milieu et les outils agissant comme médium dans l'apprentissage et le développement des compétences. Les pratiques observées soulignent que l'apprentissage médié par les technologies informatiques en milieu informel se réalise selon un processus d'autoformation, de collaboration, de coopération, d'interculturalité, de production et de fabrication.

Mots-clés : apprentissage informel, économie informelle, instrumentalisation, appropriation, $\mathrm{TIC}$

\section{Abstract}

This article contributes to research on the acquisition of knowledge and skills in informal settings through the use of information and communication technologies (ICT). It presents some outcomes of an ethnographic study on the learning processes of sellers and repairers of mobile phones in Haiti. The study shows how these professionals use IT tools to acquire the knowledge and skills necessary to carry out their trade. These processes fall into two categories: (1) learning approaches based on operations to understand and use the devices, and (2) learning approaches that include the processes of appropriation and reuse of the devices. The environment and tools act as a medium for learning and skills development. The 
practices observed show that learning mediated by computer technologies in an informal environment takes place through a process of self-training, collaboration, cooperation, interculturality, production and manufacturing.

Keywords: informal learning, informal economy, instrumentalisation, appropriation, ICT

\section{INTRODUCTION}

La rapide expansion des Technologies de l'Information et de la Communication (TIC) et leur adoption à des fins de formation (Carre, 2003) transforment les conditions d'apprentissage (Leclercq, et al. , 2015). Elles créent plus d'ouverture et l'accès libre à « des dispositifs flexibles et autonomisant » (Jézégou, 2010, p. 85) pour l'enseignement et l'apprentissage. Elles offrent aux individu-es ayant accès à Internet la possibilité d'accéder à des ressources éducatives variées et diversifiées (Bates, 2018). Comme le souligne Levy (2003), "le cyberespace supporte des technologies intellectuelles qui [...] favorisent de nouvelles formes d'accès à l'information : navigation hyper documentaire, chasse au renseignement par moteur de recherche $»($ p. 1).

De ce fait, l'apprentissage peut chevaucher entre le formel, le non formel et même l'informel :

Ce qu'il faut apprendre ne peut plus être planifié ni précisément défini à l'avance. Les parcours et profils de compétences sont tous singuliers et peuvent de moins en moins se canaliser dans des programmes ou cursus valables pour tout le monde. (Levy, 2003, p. 2)

Ainsi, un-e apprenant-e peut, en dehors de ce qui était prévu, créer un groupe de discussion portant sur un objet d'étude et se connecter à d'autres sources d'informations traitant le même sujet. Clough, et al. (2008), cité par Fahalman, 2013, rapportent que les téléphones mobiles renforcent les expériences d'apprentissage informel entre technicien-es, ces derniers s'approprient des téléphones mobiles dans un contexte informel en adaptent leurs fonctionnalités de sorte à ce que les appareils répondent à leurs besoins d'apprentissage (Fahlman, 2013, p. 6). Ce changement de paradigme au niveau des formes de dispensation et d'acquisition du savoir (Bates, 2018) pose alors la nécessité d'investiguer les manifestations et mécanismes d'apprentissage par rapport à l'activité des usagères/ers.

Cette préoccupation rejoint celles d'autres chercheur-es s'intéressant à l'analyse des savoirs issus de l'expérience des utilisateurs/trices (Garrett, 2003 ; Rabardel, 1995), ou encore, aux enjeux socio-anthropologiques des technologies (Nova, 2018) ou celles de la culture de l'appropriation des technologies (Bar, et al., 2015 ; Edgerton, 2007). Cet article s'intéresse aux apprentissages réalisés à travers les manipulations et l'utilisation des TIC dans des espaces informels de travail. II s'agit de montrer comment les pratiques de vente et de réparation des appareils informatiques (téléphones mobiles, tablettes numériques, ordinateurs portables, etc.) par les technicien-nes dans le secteur économique informel en Haïti participent au 
développement de leurs compétences. L'article fera ressortir les manipulations, les expérimentations, les transformations exercées sur et avec les TIC en dehors de leur champ de conception.

\section{MÉTHODOLOGIE}

Cette étude menée entre 2017 et 2019 a adopté une démarche ethnographique en immersion dans le milieu informel de la réparation en Haïti. L'échantillon comprend 27 participant-es dont une femme, réparti-es dans plusieurs sites d'observations différents selon l'approche ethnographique itinérante multi située (Marcus, 1995). Les périodes d'observation participantes in situ furent suivies de périodes d'observations en ligne à travers des groupes WhatsApp : une démarche tirée de l'approche ethnographique de Hine (2000) dans the virtual objects of ethnography (Hine, 2000), qui permit de saisir des détails sur les comportements de ces personnes dans l'usage des réseaux sociaux, et aussi sur le langage utilisé dans les partages de connaissances et les processus interprétatifs (Becker, 1996). Les données tirées lors des observations furent croisées à celles collectées sur les groupes WhatsApp et associées aux explications fournies lors des entretiens. Le tableau suivant synthétise les différents procédés qui ont été utilisés pour collecter les données sur le terrain.

Tableau 1. Synthèse des méthodes et types de données collectées de 2017 à 2019

\begin{tabular}{|c|c|c|c|}
\hline Méthodes & Justificatif & Outils & $\begin{array}{l}\text { Types de } \\
\text { données }\end{array}$ \\
\hline $\begin{array}{l}\text { Observation } \\
\text { participante }\end{array}$ & $\begin{array}{l}\text { Observer les usages du téléphone } \\
\text { portable par les technicien-nes les } \\
\text { interactions sociales, les modes } \\
\text { d'organisations, les connexions qui } \\
\text { existent entre les différents sites, les } \\
\text { conversations et saisir le sens donné au } \\
\text { travail qui se réalise. }\end{array}$ & $\begin{array}{l}\text { - Grilles } \\
\text { d'observations } \\
\text { - Enregistrements } \\
\text { vidéo et Photos } \\
\text { - Journal }\end{array}$ & $\begin{array}{l}\text { Textes } \\
\text { Images } \\
\text { Sons } \\
\text { Vidéos }\end{array}$ \\
\hline $\begin{array}{l}\text { Observation } \\
\text { en ligne }\end{array}$ & $\begin{array}{l}\text { Observer } 1 \text { ) les modes de partage de } \\
\text { connaissances ; 2) les intérêts pour les } \\
\text { formations ; 3) les modes d'interactions } \\
\text { sociales sur le réseau social et 4) les } \\
\text { réseautages. }\end{array}$ & - WhatsApp & $\begin{array}{l}\text { Textes } \\
\text { Images } \\
\text { Sons }\end{array}$ \\
\hline Entretiens & $\begin{array}{l}\text { Comprendre les modes d'organisation, } \\
\text { le déroulement des activités, les tâches } \\
\text { des professionnel-les, leur intégration sur } \\
\text { le marché, leurs interactions avec le } \\
\text { milieu et leurs trajectoires } \\
\text { professionnelles et académique. }\end{array}$ & $\begin{array}{l}\text { - Entretiens semis } \\
\text { dirigés } \\
\text { - Entretiens ouverts }\end{array}$ & Textes \\
\hline
\end{tabular}

Nous utilisons la méthode d'analyse thématique (Braun \& Clarke, 2006). À cet effet, il fut repéré les segments des discours connectés aux indicateurs tirés du cadre théorique (theoretical 
thematic analysis) et ceux issus des significations données à l'objet d'étude par les participantes (data-driven thematic analysis). L'intégralité des entretiens audio et individuels furent considérées comme les données principales et transcrits et codés sur Atlas.Ti. Les autres données WhatsApp, vidéos, notes de terrain et images furent ajoutées au besoin pour la description, l'interprétation ou supports aux citations. Un résumé de chaque codage fut dressé et inséré dans une matrice pour l'analyse thématique descriptive et interprétative : construction de "patterns, themes [...] making contrasts or comparison [...] building a logical chain of evidence and making conceptual/theorical coherence " (Miles, et al., 2014). En ce sens, il fut opéré un mouvement constant entre les extraits codés, les éléments de données tirés du matériau de recherche et les questions tirées des trois axes théoriques de cette recherche pour une première démarche de leur interprétation (Figure 1). II s'agit de 1 - la théorie instrumentale de Rabardel (1995) ; 2- la théorie des communautés de pratique Wenger (1998) et 3- les théories de développement et d'évaluation des compétences de Le Boterf (1998) et de Dominicé, et al. (1999).

Figure 1. Opérationnalisation des données pour l'analyse thématique

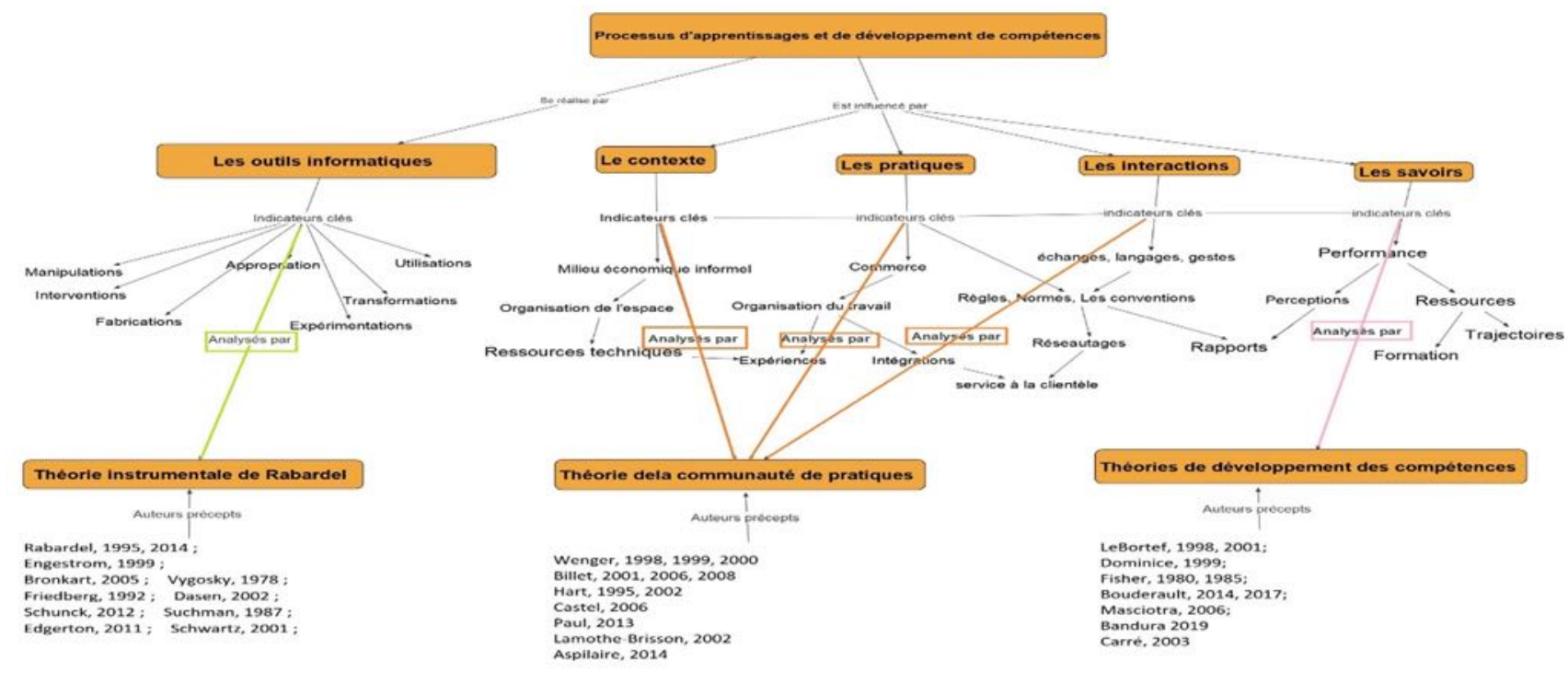

L'analyse thématique descriptive et interprétative des données permit également de rendre compte des actions, interactions, des modes d'organisations et des trajectoires qui influencent l'apprentissage et le développement de compétences des acteurs et actrices du secteur économique informel de la vente et de la réparation des appareils informatiques en Haïti. Pour garder la précision du propos, il ne sera mentionné ici que les résultats portant sur les l'analyse des processus d'appropriation des appareils informatiques dans le milieu informel, les interventions des technicien-nes pour en comprendre leur fonctionnement, ainsi que leur part joué dans la construction et la validation de leurs actions. 


\section{LE SECTEUR ÉCONOMIQUE INFORMEL DE LA VENTE ET DE LA RÉPARATION DES TECHNOLOGIES INFORMATIQUES EN HAÏTI}

La notion de "secteur informel » ou " d'économie informelle " dont Keith Hart fait mention pour la première fois en 1973 met en évidence le développement de pratiques économiques dans des sociétés "non développées ", ceci en opposition avec les règles du monde occidental (Hart, 2002). Lautier, De Miras et Morice (1991), cités par Cheneau-Loquay (2008), définissent l'économie informelle " comme les actes (ou ensemble d'actes) économiques marchands qui échappent aux normes légales en matière fiscale, sociale, juridique ou d'enregistrement statistique » (p. 110). On retrouve ces deux dimensions en Haïti, notamment avec l'arrivée des téléphones mobiles par des opérateurs/trices de télécommunication privé-es qui ont donné naissance à une prolifération d'activités nouvelles dans la vente et la réparation.

Les activités du secteur informel offrent à un grand nombre la possibilité d'avoir accès à l'outil de communication tout en assurant leur maintenance. Elles " se construisent la plupart du temps sans les fabricants eux-mêmes qui se sont longtemps désintéressés de telles pratiques » (Nova, 2018, p. 242). Les communautés de réparateurs et réparatrices passent, généralement, par des méthodes non agrées telles que l'utilisation de matériels rudimentaires, d'objets de rebus, de logiciels de piratage, de blogs de hackers, etc. pour réparer les appareils dysfonctionnels et augmenter leur durée de vie. Leurs pratiques "plus ou moins officieuses [relèvent] d'un équilibre entre des techniques très fines relevant plus de l'artisanat que d'un processus industriel bien défini " (Nova, 2018, p. 238). En somme, des communautés alternatives se constituent où l'on retrouve des technicien-nes indépendant-es qui travaillent dans l'absence de connaissances spécifiques sur les machines ou les artefacts qu'ils réparent [ou qu'ils vendent] (Houston, 2019).

Au-delà de l'apport économique qu'elles génèrent, les activités informelles de vente et de réparation en Haïti représentent aussi des situations d'apprentissage pour comprendre les fonctionnalités et le fonctionnement des appareils de télécommunication. Elles se réalisent par le biais d'actions collaboratives ou individuelles et autonomes, et comprennent des actions opératoires (tests, manipulations, dépannage, démontage, transformations, modifications). Dans ce milieu, les appareils informatiques, particulièrement le téléphone mobile sont utilisés comme médiums pour apprendre le métier, pour développer de nouvelles compétences, et comme accessoires pour des activités de revenu et de networking. L'analyse des différentes manipulations exercées sur et avec ces appareils dans le secteur économique informel peut servir à comprendre comment se mettent en place des apprentissages supportés par les NTIC en situation informelle. 


\section{INSTRUMENTALISATION DES OUTILS TECHNOLOGIQUES}

La plupart des travaux menés dans le cadre de l'instrumentalisation des outils technologiques rapportent des incidences et des transformations sur le cognitif, l'affectif et le comportement des usagères/ers (Cahour, et al., 2007). Pour Rabardel (2014), se basant sur Vygotski (1930), l'activité humaine médiatisée par un artefact conditionne son développement, transforme ses rapports au monde et ses fonctions psychologiques, selon trois logiques. Tout d'abord, la logique de fonctionnement comprend les activités de manipulation et de compréhension de l'instrument ; ensuite, les interventions sur l'instrument, les activités d'instrumentalisation et de transformation relèvent de la logique de processus ; enfin, la logique de l'utilisation comprend la réalisation des tâches productives où l'individu-e instrumenté-e produit les conditions et les moyens nécessaires pour atteindre l'objectif de sa production et valider son action. Cette théorie comprend une modélisation triadique de relations et d'interactions entre différents pôles : il s'agit des interactions entre le sujet et l'instrument (SI), entre l'instrument et l'objet sur lequel il permet d'agir (IO), entre le sujet et l'objet (S-Od) et enfin les interactions entre le sujet et l'objet médiées par l'instrument (S-Om) (Figure 2). De plus, cet ensemble est plongé dans un environnement spécifique constitué par un ensemble de conditions dont le sujet doit tenir compte dans son activité finalisée.

Figure 2. Théorie instrumentale de Rabardel (1995)

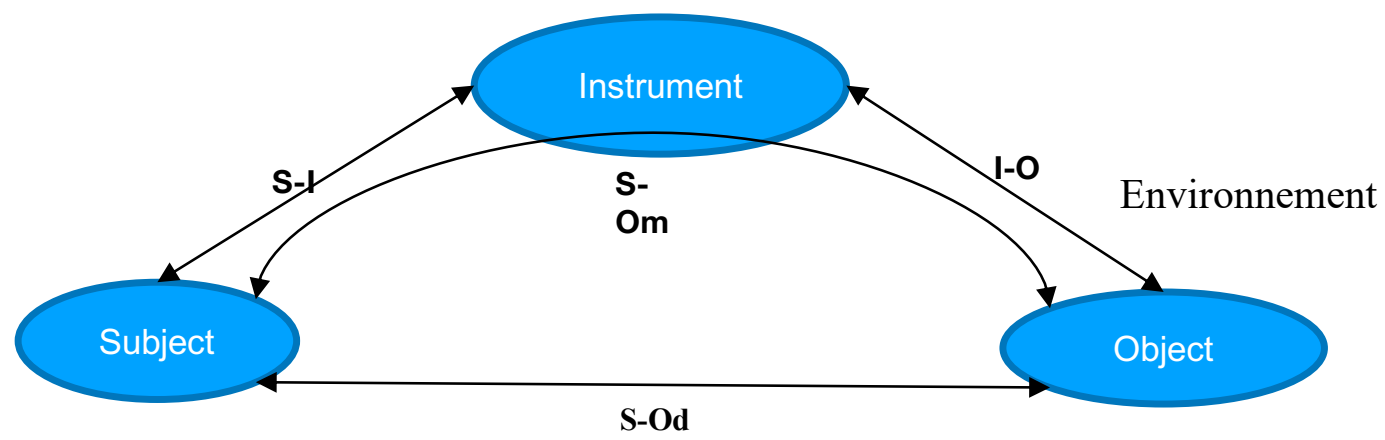

Au-delà de l'apport économique qu'elles génèrent, les activités informelles de vente et de réparationL'activité n'est pas seulement induite par les instruments, mais se réalise en fonction des besoins de la réparatrice ou du réparateur, et des conditions dans lesquelles elle se réalise. Selon Bar, et al. (2015), l'utilisation des technologies en dehors de leurs champs de conception se fait par la "réinvention » de ces technologies de sorte qu'elles répondent mieux aux besoins et aux pratiques sociales des utilisateurs/trices. Ce processus qualifié d'appropriation revient à expérimenter l'appareil technologique, à le modifier pour qu'il s'adapte au milieu dans lequel il est inséré. II correspond au fait de s'approprier de ce qui vient d'un milieu différent (Bar, et al., 2015). Plus loin les auteur-es ajoutent que le processus d'appropriation d'une nouvelle technologie dans un contexte social modifie, à la fois, la manière dont la technologie est utilisée et le cadre social dans lequel il est utilisé (Bar, et al., 2015). 
Nova (2018) souligne que les pratiques de réparation correspondent à des activités de réappropriation des techniques ou de leur fonctionnement, et avance qu'elles se réalisent dans des espaces singuliers tels que les « hackerspaces » et les "fablabs » tout autant réinventés puisque non industrialisés (Nova \& Bloch, 2020) :

La capacité à entretenir ou à remettre à neuf des objets techniques implique des talents spécifiques qui sont la plupart du temps supérieurs à ceux mis en jeu dans l'utilisation de ceux-ci. D'où la rareté des personnes capables de s'en occuper. (Edgerton, 2007, cité par Nova \& Bloch, 2020)

Notre analyse des activités des techniciens du secteur économique informel en Haïti s'est d'abord portée sur les manipulations exercées sur les appareils informatiques pour en comprendre le fonctionnement, car, selon Rabardel (1995) «l'origine de la connaissance est à chercher dans l'action » (p. 26). En d'autres termes, il s'agit de déceler dans l'action qui se produit le savoir qui la guide. De plus, les activités de transformations, de modifications, etc. exercées sur et avec les appareils sont analysées pour démontrer comment les technicien-nes passent d'une simple compréhension des outils à leur appropriation.

\section{LES ACTIVITÉS D'APPRENTISSAGES}

Dans le milieu informel de la vente et de la réparation des appareils informatiques, l'apprentissage se situe au cœur des activités professionnelles. II se produit lors des actions conduites pour comprendre le fonctionnement d'un appareil (manipulations, tests, expérimentations, etc.) ou orientées vers l'utilisation des outils informatiques et la rentabilisation de l'activité instrumentée. II peut se produire par incident (Dasen, 2002) au cours d'une observation fortuite ou de manière intentionnelle comme dans une collaboration entre maitre et apprenti : «Ce qu'on ne sait pas, il faut l'apprendre de quelqu'un d'autre » précisent-elles/ils dans le secteur de la réparation. Ici, dans l'analyse des activités d'instrumentation des appareils, il sera mis en exergue les démarches personnelles entrepris par les technicien-nes pour accéder aux savoirs nécessaires à l'exercice de leurs métiers.

\subsection{Choix et orientations pour se former}

Les personnes du milieu désirant acquérir de nouvelles connaissances le font parfois à travers des formations. Ces démarches d'apprentissages sont intentionnelles et conscientes (Schugurensky, 2007) car elles obéissent aux volontés d'apprendre des technicien-nes et dépendent des objectifs d'apprentissages qu'elles/ils se sont fixé-es. Comme le montre la figure 3, les formations proposées dans le milieu sont diverses et portent généralement sur les logiciels et les techniques de dépannage et de réparation des téléphones mobiles. Elles comprennent aussi la création de sites web, la sérigraphie, l'infographie, l'informatique bureautique, et, dans une faible mesure, l'apprentissage de l'anglais, le marketing et la comptabilité (Figure 3). 


\section{Figure 3. Exemples de séminaires de formation proposés sur les groupes de discussion WhatsApp}

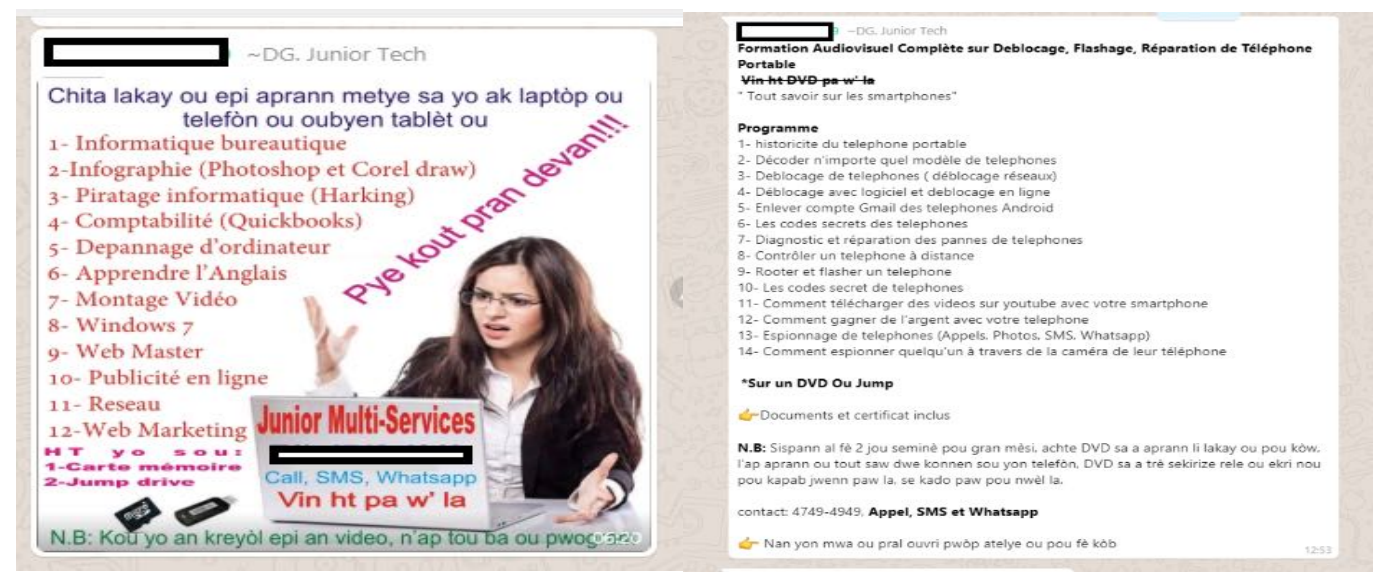

Selon leurs besoins de formation, leurs disponibilités et ressources, les technicien-nes s'orientent vers une des multiples sources de formations (workshops, centres de formation, Internet, technicien-nes pair-es...). Le but poursuivi n'étant pas tant de recevoir un diplôme ou un certificat, l'accent sera principalement mis sur le contenu des séances. Cette position se base généralement sur une analyse de coût et d'opportunité : quand le manque à gagner pour recevoir une formation est trop élevé, le choix se porte sur la poursuite de l'activité professionnelle plutôt que sur l'activité de formation. BaptisteP25, un des participants à la recherche relate cela à travers son expérience de formation avec une compagnie de formation :

Entre 2007 - 2008, le président X fit venir un grand bus pour nous emmener à un centre recevoir une formation sur la réparation des téléphones mobiles. La formation dura entre 6 à 12 mois et à la fin un certificat nous fut délivré par le CONATEL ... Je n'avais pas suivi toute la formation. J'avais estimé que c'était trop long. Cela prenait sur mon temps de travail et je ne faisais pas d'argent entre temps. Je le regrette à présent. (Baptiste, P25)

\subsection{Usage de ressources numériques}

Pour que les démarches d'apprentissage personnalisées du métier se réalisent efficacement, la connexion au Net représente une ressource nécessaire :

À cause de mon travail je ne peux pas passer un jour sans acheter des mégabits pour au moins 18 gourdes pour avoir 1 JGA. Mwen pa ka fè yon plan internet global paske mwen pa gen moyen ${ }^{1}$. Mais je ne peux passer un jour sans le net. L'internet est une nécessité. (Marc, P18)

Internet offre en effet un «paquet d'informations» que le ou la technicien-ne exploite quotidiennement pour se former et améliorer ses pratiques. AugustinP09, l'un des participants décrit son expérience avec le Net pour découvrir les fonctionnements des nouveaux modèles et leurs techniques de réparation :

\footnotetext{
${ }^{1}$ Je peux ne pas faire un abonnement global parce que je n'ai pas les moyens.
} 
Je suis déjà au courant parce qu'une fois le téléphone venu, je fais des recherches sur comment je dois l'ouvrir, comment je dois procéder. Toutes ces choses sont sur internet. C'est disponible sur YouTube... parfois vous avez besoin de faire quelque chose dans le téléphone vous ne savez pas comment l'ouvrir. Vous allez sur YouTube afin de vérifier comment on fait pour ouvrir ce modèle. Le blanc vous donne la vidéo et vous explicite comment faire. C'est très facile à faire il y avait un téléphone, j'avais un téléphone, un Galaxy, Galaxy S6 pour l'ouvrir il fallait chauffer le cache-arrière afin de trouver les vis. Je ne savais pas encore. J'ai fait des recherches sur Google et rapidement j'ai vu la vidéo et je l'ai vite fait. Le client n'a même pas remarqué cela. Je suis allé sous la table j'ai fait un click. (Augustin, P09)

Les forums de discussions sont aussi largement utilisés pour faire remonter les informations nécessaires sur les réparations. Mis à part WhatsApp, Instagram, YouTube et Facebook, les autres plateformes de discussions citées par les technicien-nes pour les recherches en ligne sont 3Tools, Comment ça marche, GSM, et aussi des «open-classroom »:

Ou gen des sites special ou se ou menm ki guide tete ou. Gen oun pakèt site, Men sa mwen konn utilise a gen rapport avec Iphone. Li rele 3Tools. Se pou Iphone. Sou google mwen just ale sou n'importe site mwen jwenn. Youn nan forum ke mwen utilise rele GSM Comment ça marche. Li vraiment intéressant. Siw gen yon problèm, ou gen yon kesyon ou gendwa pozel et puis participant yo, si yo te fait face ak problèm nan yo diw men koman pou résoud li. Li vraiment intéressant. Li pas seulement pour réparation cellulaire. Li pou dépannage ordinateurs tou ${ }^{2}$. (MT, P21)

L'usage de l'internet, des plateformes de discussion pour chercher des informations revient à opérer de nombreuses actions: prendre contact avec un spécialiste, collecter, filtrer des informations et les enregistrer (Figure 4). Certains profitent également de ces espaces pour faire la promotion de leurs produits ou encore de séminaires de formations (Figure 5).

\footnotetext{
${ }^{2}$ Vous avez des sites spéciaux où vous êtes votre guide. II y a beaucoup de sites, mais ceux que j'utilise sont liées à l'iPhone. Cela s'appelle 3Tools, c'est pour les iPhones. Sur Google, je vais simplement sur n'importe quel site que je trouve. Un forum que j'utilise s'appelle GSM comment ça marche. II est très intéressant. S'il y a un problème, vous avez une question que vous pouvez la et les participant-es, s'ils sont confronté-es au problème, elles/ils vous diront comment le résoudre. II convient non seulement pour la réparation des cellulaires, mais aussi pour dépanner les ordinateurs.
} 
Figure 4. Usage de WhatsApp pour proposer des séminaires de formation

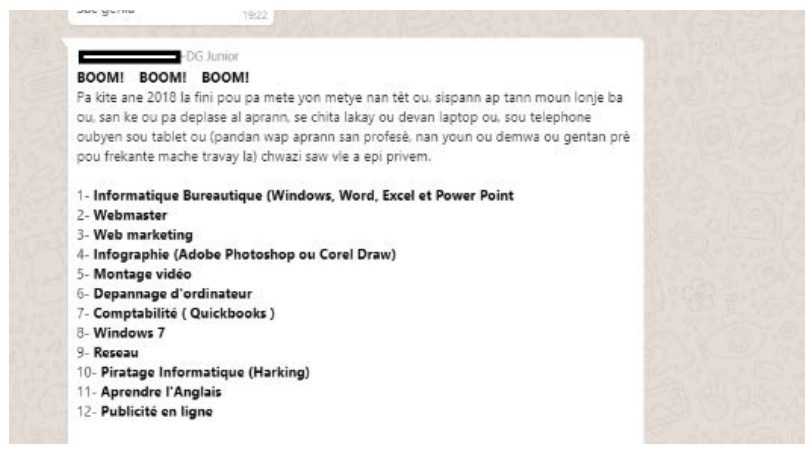

Figure 5. Usage de WhatsApp pour répondre aux besoins des clients

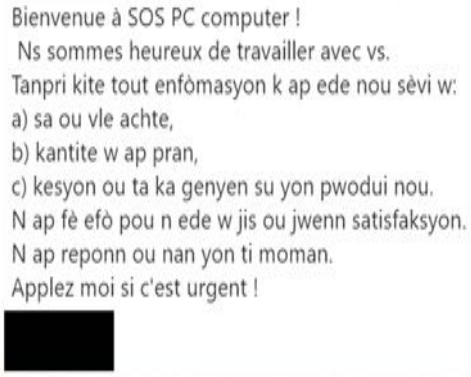

L'usage du Net permet également aux technicien-nes de maintenir leurs relations de pouvoir et de régulation sur leur apprentissage : ils savent qu'elles/ils doivent bien énoncer la situation problème et leur compréhension pour trouver les réponses appropriées en ligne, donc maitriser à la fois les techniques procédurales et explicatives : " II faut aller sur l'internet et poser les questions appropriées. Même les détails sont importants. Car une virgule peut conduire à une autre réponse. II faut donc être précis dans les questions » (MT, P21). Ce participant affirme qu'il n'y a pas de pannes qui ne peut être résolue à partir de l'usage du Net. II suffit de savoir comment mener ses recherches :

Par exemple parfois vous faites face à des réparations... par exemple vous réparez des trucs, pour vous, il n'y a pas vraiment de solution. Pourtant ce n'est pas le cas, vous faites des recherches. Vous allez sur Google, j'explique le problème, je trouve des forums, des gens qui expliquent comment ils ont surmonté telle difficulté, comment ils sont parvenus à résoudre tel problème. (Jamesbond, P13)

Internet est un outil nécessaire pour supporter l'apprentissage en temps réel, de manière instantanée, et il permet de court-circuiter des cursus de formation parfois onéreux « uniformes

\footnotetext{
${ }^{3}$ Veuillez nous laisser toute information qui nous aidera à vous servir. Ce que vous désirez acheter. La quantité que vous prendrez. Toutes les questions que vous auriez sur nos produits. Nous mettrons tout en œuvre pour vous aider à obtenir votre satisfaction. Nous vous appellerons dans un instant. Appelez-moi si c'est urgent.
} 
ou rigides qui ne correspondent pas à leurs besoins réels et à la spécificité de leurs trajets de vie » (Levy, 2003, p. 14) :

Quand vous surfez sur la toile, vous n'avez qu'à faire une commande. Demandez un livre pdf. Même si vous demandez la version gratuite c'est possible. C'est encore mieux si vous demandez à payer pour une connaissance plus approfondie. Donc, toutes ces choses vous les avez et vous les lisez ensuite vous essayez de lire entre les lignes aussi... je me suis dit si j'ai un téléphone androïde et que je peux venir observer alors il me manque quoi ? je n'ai qu'à faire une recharge minimale de dix (10) gourdes ensuite faire un plan, aller sur Google ou sur n'importe quel autre moteur de recherche ensuite taper : introduction à telle science, format pdf, téléchargement gratuit, et on vous donne le document. (Rodrigue, P04)

Ces démarches d'apprentissages soulignent une "mutation dans le sens d'un besoin de diversification et de personnalisation » (Levy, 2003, p. 14). Cette capacité à l'autoformation leur confère une liberté d'action non seulement dans le choix de leur apprentissage, mais également sur le contenu, le pilotage et la régulation de leur apprentissage.

\subsection{Expérimentations - Résolution de problèmes}

Lors des observations des différentes interventions des professionnel-les sur et avec les appareils informatiques, il fut constaté que l'apprentissage se réalise principalement par essais et erreurs, puis remédiation à travers différentes activités d'expérimentation. Les professionnelles se réfèrent aux connaissances préalables qu'elles/ils ont du métier pour identifier les erreurs, les conditions dans lesquelles elles occurrent et les corrigent. Au cours de la démarche de réparation, le réparateur ou la réparatrice manipule le modèle téléphonique pour comprendre le fonctionnement du matériel et identifier le problème de dysfonctionnement. Ces manipulations déclencheront un enchaînement d'actions qui conduiront à cibler l'opération exacte (action - résultat) permettant de résoudre le problème.

Rodrigue, un jeune apprenti, explique que pour réparer un appareil il prend le temps de le manipuler pour le connaitre et ensuite il procède à une série de tests de base qui constituent en sorte à un diagnostic :

Avant de commencer toute chose c'est d'abord la présentation de la machine, faire connaissance avec la machine à partir de sa surface afin de détecter sa marque, quelle compagnie qui l'a faite, quelques informations sur son processeur qui vous font savoir comment la manipuler. Vous faites un premier test. Après avoir fait sa connaissance, je passe à la phase de possibilités qu'il y a dans la panne. (Rogrigue, P04)

Ces manipulations sont importantes pour connaitre l'appareil et son fonctionnement, elles se réalisent généralement avec des appareils défectueux ou par abstraction ${ }^{4}$ :

\footnotetext{
${ }^{4}$ Concept que les apprentis appellent méditation
} 
Les expériences comptent beaucoup aussi et je pense que beaucoup de jeunes peuvent en témoigner... Donc, c'est ça la connaissance ... J'avais un desktop, mais j'ai décidé que ce serait mieux d'avoir un portable ... j'en ai acheté un, j'ai pratiqué un peu ce que j'ai appris. Mais c'est surtout la méditation. Parce que les pièces sont très fragiles alors on ne pas dire qu'il y a une pièce non importante pour faire de l'expérience. (Rodrigue, P04)

Une fois que le fonctionnement de l'appareil serait compris, les technicien-nes procèdent à des manipulations pour identifier la panne, la comprendre, et ensuite la réparer. Pour les travaux de manipulation, les réparations et les tests, les technicien-nes utilisent d'autres appareils comme modèles. RodrigueP04, explique, à ce propos, que certains appareils entreposés dans son atelier servent de matière première en cas de dépannage et d'autres servent à l'expérimentation (figure 6) :

Alors il y a 3 choses importantes que vous devriez savoir sur les matériels qui sont là. Premièrement il y a des commandes. Enquêtrice : hum hum. Rodrigue : deuxièmement il y a des réparations et il y a achat également. Enquêtrice : ah ok c'est ça. Rodrigue : et il y a qui ne sont là que pour les pièces. Seulement pour les pièces. (Rodrigue, P04)

\section{Figure 6. Ordinateurs portables stockés pour réparation ou pour faire des expérimentations}

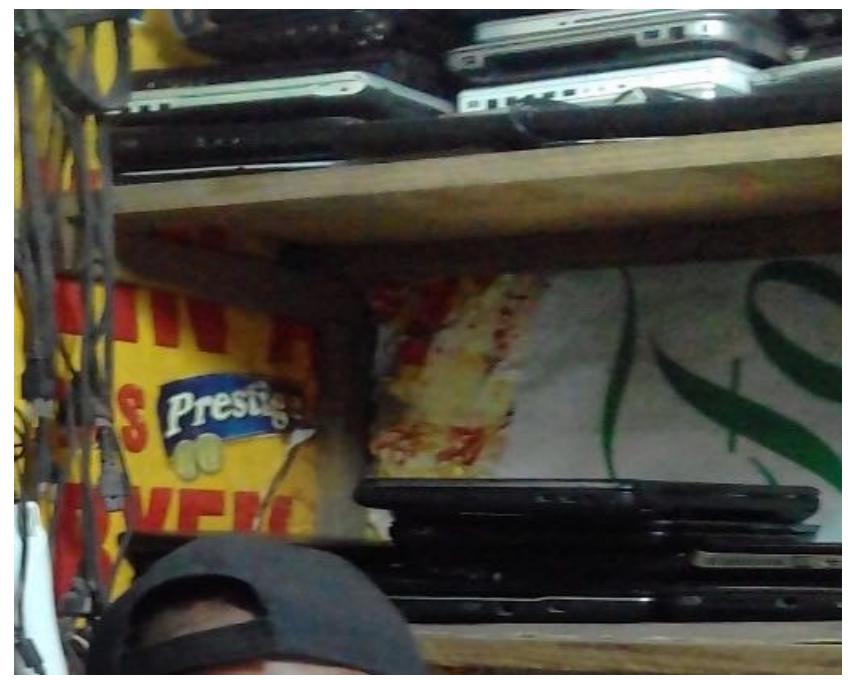

Les découvertes, ainsi que les nouvelles connaissances sur la réparation, se partagent avec la communauté comme en témoigne cette technicienne : "J'essaie toujours des expériences nouvelles et quand j'ai de bons résultats je partage avec les autres collègues » (Or, P14).

La combinaison des actions de manipulations, tests diagnostiques et expérimentations pour la compréhension du fonctionnement et de la réparation de l'appareil constitue le fondement même de l'apprentissage des technicien-nes. Les manipulations, les interventions et l'utilisation de l'instrument se réalisent simultanément ou de manière cyclique. Comme le souligne Richard (1983), cité par Rabardel (1995), « ... Iorsqu'un sujet apprend à utiliser un appareil, son objectif est en premier lieu de trouver une procédure pour atteindre le résultat qui l'intéresse. Ce n'est 
que s'il est impossible de réussir sans comprendre, qu'il s'intéresse au fonctionnement » (p. 121). Ainsi, quand la/le professionnel-le découvre qu'elle/il ne peut pas réaliser une nouvelle tâche de réparation, elle/il prend le recul qu'il faut pour découvrir le fonctionnement de l'appareil : "Si je ne parviens pas à faire telle chose je cherche à savoir d'abord comment on fait » (St Hubert, P01). Le progrès est évalué en comparant leur situation actuelle aux connaissances antérieures (Schunk, 2012).

\section{USAGES FORMELS ET INFORMELS DES OUTILS DANS LE PROCESSUS DE RÉPARATION}

Durant le processus de réparation, un objet imprévu (comme une fourchette, une bougie, un sèche-cheveux, une brosse à dents, etc.) peut être dévié de ses fonctions premières et intégrer le système d'action pour atteindre un objectif. Ces pratiques confient un pouvoir d'action et de contrôle aux technicien-nes :

Quand ces téléphones venaient d'arriver, je n'avais pas de tournevis ... c'était avec une fourchette, je ployais la fourchette, vous voyez ce que je vous dis ? Pour faire céder la vis du téléphone... Puisque c'est l'eau qui l'a pénétré dès qu'on passe un peu d'essence avec une brosse, cela enlève les petites crasses, les petites crasses où c'est rouillé. (Lucson, P16).

L'usage informel des outils s'inscrit-il comme une sous-tâche dans le processus de réparation. Par exemple, Lucson, P16, fabrique un dispositif à l'aide de fils électriques afin de diagnostiquer la panne d'écouteur d'un téléphone. Grâce à cet assemblage de fortune, il pourra vérifier si l'écouteur du téléphone est en effet en panne, et ainsi procéder à sa réparation :

Le testeur électronique, lui, vous dira si l'écouteur est endommagé. Ou bien il y a une autre façon de vérifier le problème d'écouteur ... euh vous prenez... vous mettez deux fils dans la batterie ; l'un dans le pôle positif, l'un dans le pôle négatif, vous adaptez le fils du pôle négatif dans la partie du téléphone, ensuite vous prenez l'autre du pôle positif de la batterie Enquêtrice : vous créez un testeur?

Lucson : non. Ce n'est pas un testeur. C'est pour vérifier si l'écouteur n'est pas bon ensuite vous prenez l'autre fil vous le tester, il va faire [krcrcr] si vous entendez ce bruit c'est que l'écouteur est bon. (Lucson, P16)

\section{APPROPRIATION ET RÉUTILISATION}

Dans le processus de réparation, l'activité n'est pas seulement induite par les instruments, mais se réalise en fonction des besoins des technicien-nes et des conditions dans lesquelles elle se réalise. Dans l'exécution de leurs tâches, elles/ils ne sont donc pas assujetti-es aux fonctions de l'outil, mais les transforment selon leurs connaissances du matériel, leurs besoins ou les conditions d'exécution du travail. De ce fait, un même outil comme le téléphone mobile, peut tour à tour servir d'instrument (pour visualiser une vidéo de réparation sur YouTube, ou servir de modèle pour une réparation) ou d'objet (appareil cassé qui est réparé). 
Les images de la figure 7, montrent la polyvalence de l'outil téléphonique. Sur la première image, un technicien se sert d'un téléphone, qu'on ne voit pas, comme outil pour capturer deux images d'un téléphone en panne pour envoyer une demande spécifique de matériels dans un groupe WhatsApp. La deuxième montre les deux actions combinées : en premier plan, le smartphone qu'on observe est l'objet à vendre et celui qui apparait en reflet sert de lien pour la communication sur WhatsApp.

Figure 7. Le technicien fait une demande d'écran et de torche pour réparer le téléphone (à gauche). Le technicien montre un modèle de téléphone à vendre (à droite)

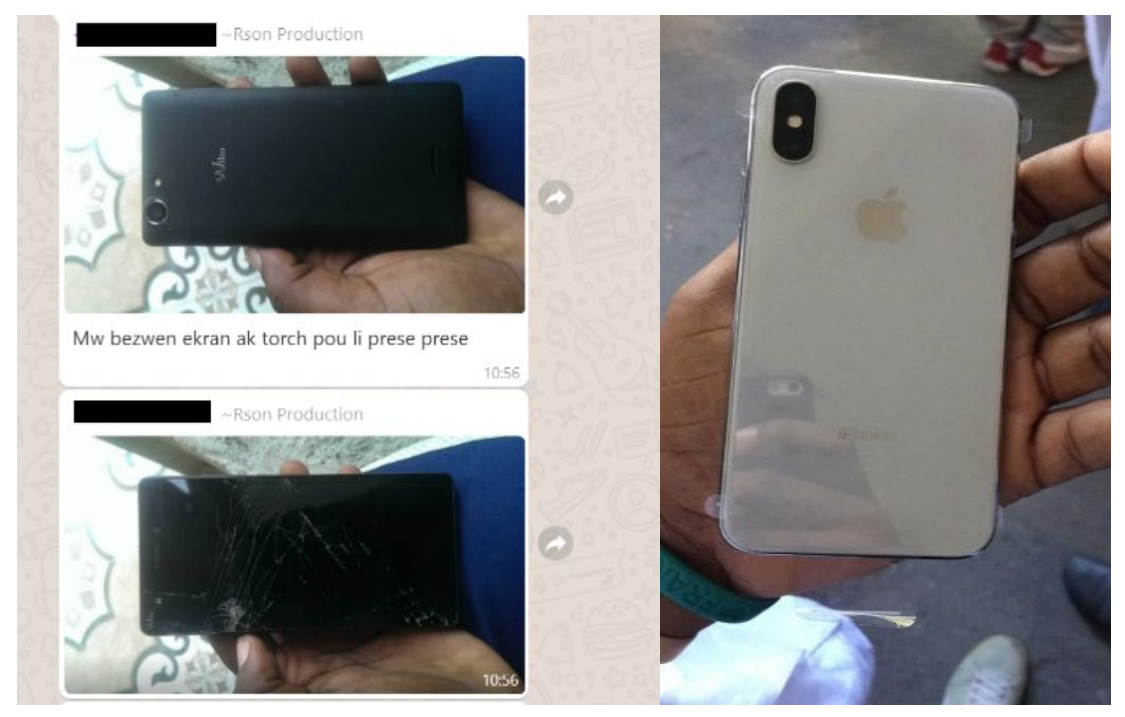

L'outil évolue donc en dehors de son champ de conception par une appropriation à travers de multiples manipulations: "Users begin experimenting with technology, exploringhow they might adapt it or adapt practices around it. Users may modify a device, download, or program new applications, invent unintended uses, or develop new practices that leverage its possibilities " ${ }^{5}$ (Bar, et al. , 2015, pp. 618-619). Ainsi, les appareils informatiques sont ouverts, craqués, bricolés, réutilisés et transformés de sorte qu'ils s'adaptent aux réalités et aux besoins des technicien-nes : «Parfois je trouve un téléphone usagé par terre, je l'ouvre, je l'examine, je trouve de bonnes pièces à l'intérieur, je les bricole et je parviens à fabriquer mon téléphone avec » (Walanmou, P03).

Cette « capacité à entretenir ou à remettre en état des objets techniques implique des talents spécifiques qui sont la plupart du temps supérieurs à ceux mis en jeu dans l'utilisation de ceuxci » (Nova, 2018, p. 237). Ces activités de réparation, de réutilisation de transformation et de modification ouvrent la voie à des pratiques innovantes - une sorte d'hybridation de pratiques et de conception - créant de nouveaux services et métiers qui sans cela n'auraient pas pu voir le jour.

\footnotetext{
${ }^{5}$ Comment elles/ils pourraient les adapter ou adapter leurs pratiques autour d'elles/eux pour que la technologie serve mieux leurs propres intérêts.
} 


\section{APPROPRIATION ET FABRICATION}

Nous avons pu observer une appropriation du téléphone mobile chez deux participants que I'on peut qualifier d'artisans et non de techniciens à cause du type de travail particulier qu'ils exercent. Ces actions correspondent à ce que l'historien des techniques, Edgerton (2007), définit comme la créolisation de la technologie, "technologies transplanted from their place of origin finding use of a greater scale elsewhere " (Edgerton, 2007, p. xiv). Nous constatons une transformation des technologies en de nouveaux modèles estimés plus adaptés aux besoins et aux réalités du milieu. Bar, et al. (2015) ajoutent que la créolisation est avant tout un processus à travers lequel les personnes réalisent un mélange et «re-mix » de culture. II va au cœur de leur affectif, de leur compréhension et de leur proposition. Les images de la figure 8 ci-contre montrent les productions de ces deux artisans : d'une part, on trouve le téléphone mobile fait en bois até plat, et de l'autre, le robot humanoïde Dumax réalisé à partir de matériels de rebus et d'un téléphone Android.

Figure 8. AtèPlat, téléphone mobile fabriqué à partir de bois (à gauche). RobotDumax2018, premier robot humanoïde en Haïti, fabriqué à partir de matériels de rebus. L'interface de communication est le téléphone (à droite)

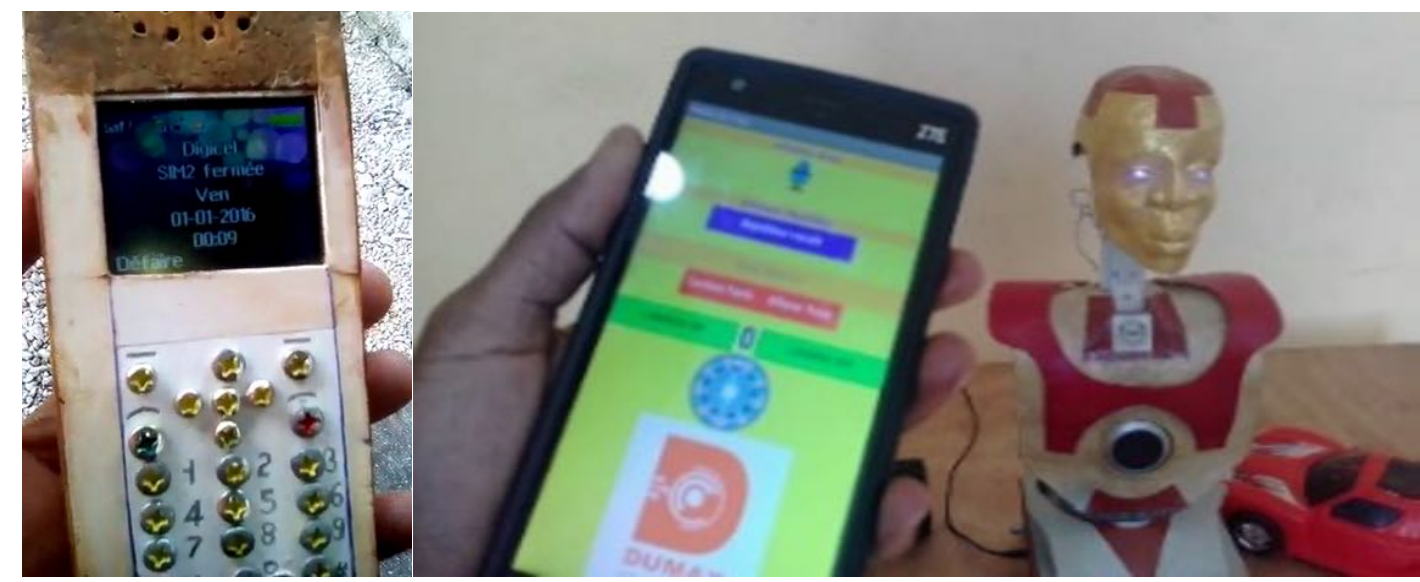

Le téléphone en bois de Walanmou possède les mêmes fonctionnalités de base qu'un téléphone Android, à savoir : faire des appels, envoyer des sms, prendre et partager des photos, etc. La transformation de l'original en un téléphone en bois est considérée innovante car elle ne s'inscrit pas uniquement dans une logique de restauration (Nova, 2018), mais aussi dans une recherche d'efficacité : l'outil est modifié, transformé pour suppléer à certaines déficiences « en [les] rendant plus productif ou plus facile " ou pour "atteindre de nouveaux objectifs ou objets qui n'auraient pu être abordés sans un changement de la situation " (Peraya \& Jaccaz, 2004, p. 3) :

Mon téléphone contient un emplacement pour carte mémoire. Voilà l'endroit où vous placez la carte mémoire. Vous pouvez constater que celle que fabrique le blanc ne prend qu'un seul casque, ça veut dire que quand j'écoute de la musique chez moi si mon enfant ou ma femme veut écouter de la musique, ils n'ont pas à me déranger en prenant mon casque. Je 
l'ai fabriquée de façon à ce qu'elle en prenne deux. J'ai mis une prise ici et une autre là. Si ce téléphone tombe sur le sol, il s'écrasera. Le mien, cependant, s'il tombe sur le sol, ne s'écrasera pas ... Ensuite, vous pouvez voir ... que le signal se trouve sur le cache-arrière. Celui du blanc est à l'intérieur, le mien est à l'extérieur. (Walanmou, P03)

On retrouve un acte d'appropriation qui se manifeste à travers le désir d'adapter l'outil à une réalité socioculturelle différente de son lieu de conception. En effet, Walanmou utilise le terme «blanc » quand il présente l'outil original pour signifier qu'il est étranger, d'une culture différente. II insiste alors sur les transformations apportées à l'outil pour qu'il s'accorde mieux à son milieu :

Enquêtrice : pourquoi l'avez-vous fabriqué ainsi ?

Walanmou : je l'ai fabriqué ainsi parce que si votre mère est à la campagne et vous, à Portau-Prince, le téléphone ne perdra pas son signal réseau. Les os des vieux ne se régénèrent pas. Parfois les vieux montent sur leur toit pour trouver du signal c'est tout ce que je peux vous dire. J'ai installé le système ainsi pour capter les ondes... Le son de mon téléphone est intense même si vous avez des troubles de l'audition. . la façon dont le mien a été conçu permet que le son soit intense. Ou wè telephone sa. Li tonbe atè li pap kraze. Kote mwen mete signal la ? SI oun moun andeyo ap pale li pap pèdi signal paske signal la pi pres pase pa blan an. Ou wè ? Se la signal la ye. Granmoun na li menm pap besoin monte yon tete morne poul al cechè signal. Lè li menm pandan lap monte poul al cheche signal la li ka tonbe. Se poutèt sa mwen mete signal la la li menm. Sa important ${ }^{6}$. (Walanmou, P03)

L'apprentissage dans ces activités de création porte également sur l'instrumentation des outils. Dumax utilise son téléphone pour apprendre la programmation et la conception informatique de manière à pouvoir construire un robot humanoïde. II agence matériaux de rebus, techniques rudimentaires, art et technologies informatiques pour créer son œuvre. Dans son atelier de travail, le téléphone lui sert à la fois de médium pour apprendre les langages de programmation, mais aussi comme interface de communication avec son robot et encore comme matériau :

Donc je fais des recherches sur eux pour voir comment je peux les acquérir et les programmer de façon autonome pour que je n'aie pas à le démarrer avec le téléphone, que le micro se comporte comme une personne vous lui posez des questions, il vous entend en temps réel et vous répond. Pour que le robot réagisse, l'interface de communication c'est le téléphone. Je me sers du téléphone parce que toute l'application est dessus... Le système robot est fait de deux façons soit que sa connaissance est déjà embarquée, ou le téléphone, vous mettez toute sa connaissance sur le téléphone ou bien vous l'hébergez de façon...c'est-à-dire si tout est embarqué sur le téléphone on n'aura pas besoin d'internet. Ensuite, j'ai re-confectionné son intérieur au niveau électronique. (Dumax, P17)

\footnotetext{
${ }^{6}$ Vous voyez ce téléphone. II est tombé au sol et ne s'est pas cassé. J'ai placé le signal là. Si quelqu'un de la province l'utilise pour parler, il ne perdra pas le signal car le signal est plus proche que le signal blanc. Vous voyez ? Voilà le signal. Le vieux n'aura plus besoin de grimper en haut d'une colline pour chercher à capter un signal. Quand il grimpe pour chercher un signal, il peut tomber. C'est pourquoi j'ai placé le signal là. C'est important.
} 
Nous observons, alors, comme le démontre la figure 9 ci-après, que lors du processus de créolisation un acte d'invention où les ressources disponibles sont organisées ou restructurées " soit par usage informel d'un outil formel, soit par élaboration d'outils informels » de manière à former « un ensemble homogène où se réalise un meilleur équilibre entre les objectifs d'économie et d'efficacité » (Rabardel, 1995, pp. 104-105) :

La seule chose importée qu'il y a à l'intérieur est le microcontrôleur qui contrôle les mouvements. L'un de mes frères revenait d'un mariage aux États-Unis j'en ai profité pour faire la commande qui m'a couté 45 euros/ \$55 us. Ok ... c'est les seules choses importées ... après je l'ai fait avec des matériaux de recyclage : du carton, du papier. Toutes les pièces je les ai prises dans des radios, des télévisions: les CD et les boites de CD, et aussi les anciennes roues de voiture de mon petit garçon pour le faire ... » Vous comprenez ? Alors j'ai amélioré mon robot ... je vous présente RobotDumax 2019, ses bras sont nouveaux. Son système de reconnaissance vocale est incrémenté. Le tout est intégré dans une tablette Android qui lui sert de visage aussi. Il peut vous permettre de voir tout ce qu'il voit à distance. (Dumax, P17)

\section{Figure 9. Robot DUMAX}

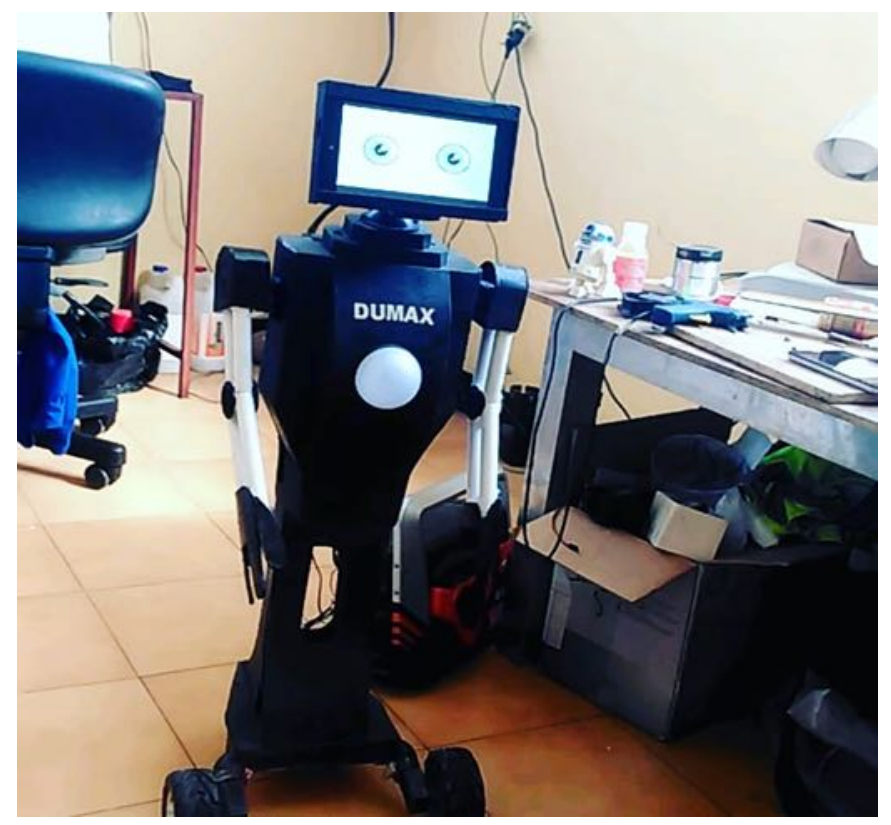

Ces actes de transformations portées sur la machine ou avec la machine proviennent également d'une curiosité personnelle pour découvrir et créer :

Je suis à la recherche de solutions. J'ai toujours dit face à un problème il y a une solution. Elle peut ne pas être la solution réelle, mais c'est une solution alternative quand même. Par exemple je peux avoir besoin d'une imprimante 3D pour imprimer la structure du robot, mais la situation économique précaire dans laquelle je vis, si j'attends l'imprimante 3D je ne finirai jamais ce que j'ai commencé, mais il y a une autre solution possible. J'ai appris à le faire. (Dumax, P17) 
Ces transformations innovantes représentent un élément imprévu dans le secteur économique informel. Elles provoquent un changement dans la représentation de la production et de l'usage des appareils en intervenant sur les produits pour en créer de nouveaux en dehors des espaces manufacturés ou industrialisés : "Ces lieux et les pratiques sont d'autant plus intéressants qu'ils se sont constitués sans forcément l'aide et la contribution des constructeurs « (Nova, 2018). Dans le processus de créolisation, les artisan-es établissent donc un changement de l'imaginaire en mettant en valeur une production libre et personnalisée d'objets en parallèle aux modèles économiques dominants.

\section{CONCLUSION}

La production des connaissances se réalise à partir des différentes actions réalisées pour appréhender les objets, comprendre leurs fonctionnalités et les réutiliser. En s'interrogeant sur les savoirs et compétences acquises des appareils informatiques et sur la manière dont ces connaissances sont acquises par les technicien-nes du secteur économique informel en Haïti, nous avons répertorié les interventions, les transformations, les relations que ces dernières/ers entretiennent avec les outils technologiques. L'ensemble de ces actions démontrent comment les technicien-nes instrumenté-es gèrent « le maintien en condition fonctionnelle de l'artefact (logique de fonctionnement) comme le processus de transformation des objets (logique de processus) pour que l'artefact soit un moyen effectif de son action (logique d'utilisation, rapport instrumental) » (Rabardel, 1995, p. 51).

L'analyse de ces activités a permis de mettre à jour les démarches d'apprentissages et d'appropriation des TIC dans le domaine de la vente et de la réparation des appareils informatiques en Haïti. Les observations des activités de réparation font état de la mobilisation de stratégies cognitives d'apprentissages (observation, traitement de l'information, expérimentation application, etc.), mais aussi de stratégies d'auto-organisation, créative et innovante. Les activités mobilisées par les technicien-nes dans l'exercice de leur métier participent donc aux processus qui qui font émerger leurs savoirs et leurs compétences. Les travaux de réparation, de vente et de fabrication des matériels informatiques en situation informelle sont assujettis aux conditions de l'environnement contextuel dans lesquels ils se réalisent. Le contexte est donc l'élément constitutif de l'acte de réparation puisque les représentations des connaissances des appareils sont incorporées aux pratiques de réparation et au contexte spécifique de réparation.

\section{RÉFÉRENCES}

Bar, F., Weber, M. S., \& Pisani, F. (2015). Mobile technology appropriation in a distant mirror: Baroquization, creolization, and cannibalism. New Media and Society, 18(4), 617-636. https://doi.org/10.1177/1461444816629474

Bates, T. (2018). Teaching in a Digital Age: Guidelines for designing teaching and learning. https://openlibrary-repo.ecampusontario.ca/jspui/handle/123456789/276 
Becker, H. (1996). The epistemology of Qualitative Research. Ethnography and Human Development: Context and Meaning in Social Inquiry, 27, 53-70.

Braun, V., \& Clarke, V. (2006). Using thematic analysis in psychology. Qualitative Research in Psychology, 3(2), 77-101. https://doi.org/10.1191/1478088706qp063oa

Cahour, B., Brassac, C., Vermersch, P., Bouraoui, J. L., Pachoud, B., \& Salembier, P. (2007). Étude de l'expérience du sujet pour l'évaluation de nouvelles technologies : L'exemple d'une communication médiée. Revue d'Anthropologie Des Connaissances, 1(1), 85-120. https://doi.org/10.3917/rac.001.0085

Carre, P. (2003). La double dimension de l'apprentissage autodirige la contribution à une theorie du sujet social apprenant. Canadian Journal for the study of Adult Education, 17(1), 66-91.

Cheneau-Loquay, A. (2008). Rôle joué par l'économie informelle dans l'appropriation des TIC en milieu urbain en Afrique de l'Ouest. Netcom, 22(1/2), 109-126. https://doi.org/10.4000/netcom.2013

Dominicé, P., Josso, M.C., Müller, R.,Pfister, M.. Rudin Equey, F,. Sthal-Thuriaux. A, \& Turkal, L. (1999). Les origines biographiques de la compétence d'apprendre. http://archiveouverte.unige.ch/unige:18575

Edgerton, D. (2007). The Shock of the Old: Technology and Global History since 1900. New York, NY: Oxford University Press.

Fahlman, D. W. (2013). Examining Informal Learning using Mobile Devices in the Healthcare Workplace / Examen de l'apprentissage informel par l'utilisation d'appareils mobiles dans le milieu des soins de santé. Canadian Journal of Learning and Technology/La Revue Canadienne de l'apprentissage et de La Technologie, 39(4). https://doi.org/10.21432/t2x59h

Garrett, J. J. (2003). The Elements of User Experience: user-Centered Design for the Web. Indianapolis, IN: New Riders.

Hart, K. (2002). Quelques confidences sur l'anthropologie du développement. http://www.ethnographiques.org/2002/Hart

Hine, C. (2000). Virtual Ethnography. Thousand Oaks, CA: SAGE publications Inc.

Houston, L. (2019). Mobile Phone Repair Knowledge in Downtown Kampala: Local and TransLocal Circulations. In I. Strebel, A. Bovet, \& P. Sormani (Eds.), Repair Work Ethnographies: Revisiting Breakdown, Relocating Materiality (pp. 129-160). Singapore: Springer.

Jézégou, A. (2010). Le dispositif GEODE pour évaluer l'ouverture d'un environnement éducatif The Journal of Distance Education/Revue de l'Education a Distance, 24(2), 83-108.

Le Boterf, G. (1998). Evaluer les compétences. Quels jugements? Quels critères? Quelles instances? Education Permanente, 135, 143-152.

Leclercq, G., \& Varga, R., (2012). Collaborer, oui mais comment? In B. Bourassa, M. Boudjaou (Eds.), Des recheches collaboratives en sciences humaines et sociales : enjeux, modalites et limites (pp.77-95) Québec, QC : Presse de l'Universite Laval.

Levy, P. (2003). Éducation et cyberculture.

http://www.ub.edu/prometheus21/articulos/obsciberprome/levy2.pdf

Marcus, G. E. (1995). Ethnography in / of the World System: The Emergence of Multi-Sited. 
Ethnography Annual Review of Anthropology, 24(1995), 95-117.

Miles, B. M., Huberman, M., \& Saldana, J. (2014). Qualitative Data Analysis A Method Sourcebook. Thousand Oaks, CA: SAGE publications Inc.

Nova, N. (2018). Figures Mobiles: une anthropologie du smartphone. Thèse de doctorat, Université de Genève.

Nova, N., \& Bloch, A. (2020). Dr. Smartphones: an ethnography of mobile phone repair shops. Lausanne: IDP.

Rabardel, P. (1995). Les hommes et les technologies. Approche cognitive des instruments contemporains. Paris : Armand Colin.

Schugurensky, D. (2007). 'Vingt mille lieues sous les mers': Addressing four challenges of informal learning [«Vingt mille lieues sous les mers»: Les quatre défis de l'apprentissage informel]. Revue Francaise de Pedagogie, 160, 13-27. https://www.scopus.com/inward/record.uri?eid=2-s2.060950274634\&partnerID=40\&md5=291b0c9e68e9b2381ab38cc3c857e54b

Schunk, D. H. (2012). Learning theories: An educational perspective. London: Pearson Education.

Wenger, E. (1998). Communities of practice: learning as a social system. Systems thinker, 9(5), 2-3.

Open Access Publications - Bibliothèque de l'Université de Genève Creative Commons Licence 4.0

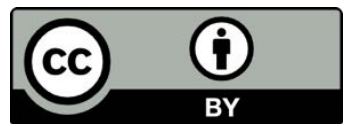

